\title{
A COMUNIDADE DE MACROINVERTEBRADOS AQUÁTICOS E CARACTERÍSTICAS LIMNOLÓGICAS DAS LAGOAS CARIOCA E DA BARRA, PARQUE ESTADUAL DO RIO DOCE, MG
}

\author{
MARQUES, M. G. S. M., ${ }^{1}$ FERREIRA, R. L. ${ }^{1}$ e BARBOSA, F. A. R. ${ }^{2}$ \\ ${ }^{1}$ Programa de Pós-graduação em Ecologia, Conservação e Manejo de Vida Silvestre, ICB/UFMG \\ ${ }^{2}$ Instituto de Ciências Biológicas, Departamento de Biologia Geral, Universidade Federal de Minas Gerais, \\ Av. Antonio Carlos, 6629, C.P. 486, CEP 31270-901, Belo Horizonte, MG
}

Correspondência para: Francisco A. R. Barbosa, Instituto de Ciências Biológicas, Departamento de Biologia Geral, Universidade Federal de Minas Gerais, Av. Antonio Carlos, 6629, C.P. 486, CEP 31270-901, Belo Horizonte, MG

Recebido em 20/08/97 - Aceito em 28/12/98 - Distribuído em 30/06/99

(Com 3 figuras)

\section{ABSTRACT \\ Macroinvertebrate community and limnological caracteristics of Lagoa Carioca and Lagoa da Barra, State Park of Rio Doce, Minas Gerais, Brazil}

The aquatic macroinvertebrate community, often used to assess water quality, was investigated in two lentic systems from among the lakes in the Middle Rio Doce, which showed different conservation status. The Lagoa da Barra and Lagoa Carioca were sampled at their margins and in the limnetic zone, collecting water for physical and chemical characterization, and sediment to obtain the benthic organisms. The Lagoa da Barra, which suffers strong impacts due to human actions, showed less oxygen concentration, higher conductivity, and its biological community showed less richness (14 taxa versus 33 in Lagoa Carioca). The analysis of fish trophic guilds suggests that the predation pressure on the macroinvertebrate community is higher in Lagoa da Barra as well. The Lagoa Carioca, besides having higher richness, showed a more well-structured community in terms of functional feeding groups, reflecting its better state of conservation, because it is inside the limits of the State Park of Rio Doce.

Key words: aquatic macroinvertebrates, conservation, lentic system.

\section{RESUMO}

A comunidade de macroinvertebrados aquáticos, freqüentemente utilizada na avaliação da qualidade de água, foi investigada em dois sistemas lênticos do Médio Rio Doce, que se apresentam sob diferentes status de conservação. As lagoas da Barra e Carioca foram amostradas em suas margens e na região limnética, retirando-se água para caracterização física e química e sedimento para coleta de organismos bentônicos. A lagoa da Barra, que vem sofrendo sucessivos impactos pela ação antrópica, mostrou teores mais baixos de oxigênio dissolvido, elevada condutividade elétrica e sua comunidade bentônica, menor riqueza (14 taxa, contra 33 presentes na lagoa Carioca). A análise das guildas tróficas da ictiofauna sugere que a pressão de predação sobre a comunidade de macroinvertebrados é também maior na lagoa da Barra. A lagoa Carioca, além de apresentar maior riqueza de taxa, mostrou uma comunidade melhor estruturada em termos de grupos funcionais de alimentação, refletindo seu melhor estado de conservação, por estar dentro dos limites do Parque Estadual do Rio Doce.

Palavras-chave: macroinvertebrados aquáticos, conservação, sistemas lênticos. 


\section{INTRODUÇÃO}

A comunidade de macroinvertebrados bentônicos é um importante componente do sedimento de rios e lagos, sendo fundamental para a dinâmica de nutrientes, a transformação de matéria e o fluxo de energia (Callisto \& Esteves, 1995). O biorrevolvimento da superfície do sedimento e a fragmentação do litter proveniente da vegetação ripária são exemplos de processos sob a responsabilidade da comunidade bentônica, que resultam na liberação de nutrientes para a água e na aeração dos sedimentos (Devái, 1990; Cummins et al., 1989), sendo a saúde e a qualidade de um corpo d'água dependentes de tais processos.

A qualidade do hábitat é um dos fatores mais importantes no sucesso de colonização e estabelecimento das comunidades biológicas em ambientes lênticos ou lóticos. A flora e a fauna presentes em um sistema aquático são também influenciadas pelo ambiente físico do corpo d'água (geomorfologia, velocidade da corrente, vazão, tipo de substrato, tempo de retenção). Estando a situação de um corpo d'água estreitamente relacionada às atividades humanas realizadas à sua volta, o primeiro passo para a compreensão de como as comunidades de macroinvertebrados bentônicos estão reagindo à alteração da qualidade de água é identificar quais variáveis físicas, químicas e biológicas estão afetando os organismos (Tate \& Heiny, 1995).

Este trabalho tem por objetivos: i) determinar algumas das características limnológicas de duas lagoas (Barra e Carioca), que se apresentam sob diferentes usos e estados de conservação; ii) comparar a comunidade de macroinvertebrados bentônicos, em termos de grupos funcionais de alimentação; e iii) avaliar a estruturação desta comunidade com base nas guildas tróficas de peixes.

\section{ÁREA DE ESTUDO}

A lagoa Carioca está situada dentro dos limites do Parque Estadual do Rio Doce (PERD); é considerada uma lagoa rasa (profundidade máxima: $11,8 \mathrm{~m}$; média: $2,7 \mathrm{~m}$ ), de formato arredondado (comprimento máximo: 576,3 m; largura máxima: 329,8 m), alcançando uma área de 13,2 ha (Barbosa \& Tundisi, 1980). Trata-se de um ambiente razoavelmente bem estudado e representativo de lagos moderadamente eutrofizados do PERD.
Por outro lado, não existem dados na literatura sobre a lagoa da Barra. Trata-se de um sistema natural, bem maior que a lagoa Carioca; possuindo formato dendrítico, localizada fora dos limites do Parque, tendo como vegetação predominante em sua bacia uma antiga plantação de Eucalyptus spp., hoje sem qualquer manejo. Esse ambiente vem sendo utilizado para a pesca recreativa com bastante intensidade.

\section{MATERIAIS E MÉTODOS}

Este estudo foi desenvolvido em julho de 1996, durante o inverno, período característico de seca na região.

A partir de mapas do IBGE (escala 1:100.000) e de acordo com a metodologia proposta por Cole (1983) foi estimado o índice de desenvolvimento das margens para as duas lagoas.

Selecionou-se dois pontos de amostragem em cada lagoa: um na região profundal e outro na região litorânea. Em ambos os pontos foram retiradas amostras de água e sedimentos para análises. Foram determinadas as seguintes variáveis in situ: temperatura, $\mathrm{pH}$ e condutividade elétrica e, em laboratório, foram determinados os teores de oxigênio dissolvido (Winkler, 1888, modificado) e alcalinidade total de acordo com Mackreth et al. (1978).

Amostras de sedimentos foram coletadas em ambas as lagoas, utilizando-se uma draga de Eckman-Birge, com a qual retiraram-se três amostras, tanto na região litorâna quanto na zona profundal, e mais 50 amostras na zona limnética através de conchas metálicas de fundo perfurado (abertura $<1 \mathrm{~mm}$ ).

Os organismos encontrados foram identificados em morfo-espécies. Calculou-se a diversidade de cada ambiente através do índice de ShannonWiener (ln) e a diferença entre a diversidade das lagoas foi testada pelo teste $t$ de Hutcheson.

As guildas tróficas de peixes foram analisadas a partir de trabalhos realizados simultaneamente no local e de dados da literatura (Vieira, 1994).

\section{RESULTADOS E DISCUSSÃO}

As principais características físicas e químicas das duas lagoas são mostradas na Fig. 1. O índice de desenvolvimento das margens estimado para a lagoa Carioca foi 1,12, enquanto para a lagoa da Barra esta estimativa foi muito maior, alcan- 
çando um valor de 4,45. O perímetro e a área da lagoa da Barra foram, respectivamente, de 19,7 $\mathrm{km}$ e $1,55 \mathrm{~km}^{2}$. As mesmas variáveis estimadas para a lagoa Carioca foram de $1,47 \mathrm{~km} \mathrm{e} 0,13 \mathrm{~km}^{2}$.

Nenhum dos ambientes encontra-se termicamente estratificado, sendo lagos monomíticos quentes, apresentando variações diurnas pronunciadas, particularmente no verão, como definido para a lagoa Carioca por Barbosa \& Tundisi (1989), e características de lagos tropicais, conforme salientado por Esteves (1988).

$\mathrm{Na}$ maioria dos casos, a estratificação térmica condiciona a estratificação química, mas em lagos de regiões tropicais esta (especialmente de oxigênio dissolvido) independe da térmica.

Assim, a lagoa da Barra, por exemplo, apresenta um perfil de oxigênio do tipo ortogrado, enquanto a Carioca apresenta um perfil do tipo clinogrado.

A alcalinidade total não apresentou grandes variações dentro de cada lagoa. Entretanto, a lagoa da Barra apresentou maior concentração de bicarbonatos $(2,45 \mathrm{mmol} / \mathrm{L}$, contra $1,20 \mathrm{mmol} / \mathrm{L}$ na lagoa Carioca), indicando maior disponibilidade de carbono para os produtores primários. A condutividade elétrica apresentou-se mais elevada na lagoa da Barra, demonstrando uma maior concentração de íons dissolvidos.

A comunidade bentônica foi mais rica e abundante na lagoa Carioca (Tabela 1). Foram encontrados 33 taxa de macroinvertebrados na lagoa Carioca e apenas 14 na lagoa da Barra. A diversidade estimada pelo índice de Shannon-Wiener foi de 0,70 e 0,69 nas lagoas Carioca e Barra, respectivamente (diferença não significativa pelo teste $t$ de Hutcheson). Foi encontrado um número total de organismos igual a 1.112 e 109 indivíduos nas lagoas Carioca e Barra, respectivamente. Chironomidae foi o táxon numericamente predominante em ambas, tendo sido encontrada apenas a tribo Chironomini, pertencente à subfamília Chironominae. Segundo Di Giovanni et al. (1996), a família Chironomidae quase sempre apresenta-se como dominante, tanto em ambientes lóticos como lênticos, devido à sua tolerância a situações extremas como hipóxia e grande capacidade competitiva.

Em nenhum dos ambientes foram observados bancos de macrófitas na região litorânea, na lagoa Carioca encontraram-se apenas alguns exem- plares esparsos da macrófita flutuante Salvinia spp. Entretanto, as duas lagoas apresentaram uma fauna bentônica mais rica e abundante na região litorânea, enquanto na região profundal a fauna sobre o sedimento restringiu-se a poucos grupos, notadamente os dípteros Chironomidae e Chaoboridae e anelídeos da classe Oligochaeta.

Em levantamentos bentofaunísticos realizados por Fukuara et al. (1987) nas lagoas Jacaré e D. Helvécio, também pertencentes ao PERD, observou-se que a família Chaoboridae (Diptera) dominava a comunidade, tanto em termos numéricos (densidade) quanto em relação à biomassa, excetuando-se a região litorânea, na qual Chironomidae apresentava-se em maior abundância. No presente trabalho, a família Chaoboridae foi de pequena participação na composição da comunidade, sendo encontrada apenas uma morfo-espécie representada por poucos indivíduos na região profunda de cada ambiente.

Alguns dos organismos encontrados neste estudo não são necessariamente bentônicos, e sua presença nas amostras deve-se à contaminação das mesmas. Assim, tem-se a família Nabidae que é de hábito terrestre e, provavelmente, encontravase na vegetação junto às margens da lagoa no momento da coleta. Outras famílias como Gerridae, que pertence ao nêuston, e Notonectidae, que é livre natante, também foram capturadas nas amostras, mas sua presença pode ser considerada acidental. As três famílias da ordem Coleoptera encontradas neste estudo foram representadas apenas por indivíduos adultos, não sendo encontradas formas imaturas.

Segundo a classificação de grupos funcionais de alimentação de Merrit \& Cummins (1984), os organismos encontrados foram agrupados em seis guildas funcionais: predadores, filtradores, fragmentadores, raspadores, coletores e parasitas. A contribuição de cada grupo para a composição da comunidade é mostrada na Fig. 2. A dominância de organismos coletores, principalmente Chironomidae, indica um enriquecimento de matéria orgânica no sedimento (Dévai, 1990). Entretanto, a presença de peixes detritívoros na lagoa Carioca significa competição por recursos (matéria orgânica sedimentada) para os organismos coletores, explicando assim a maior abundância dos mesmos na lagoa da Barra, na qual não ocorrem peixes de tal grupo trófico (Fig. 3). 
TABELA 1

Organismos encontrados nas regiões litorâneas e limnéticas das lagoas Carioca e da Barra, PERD, MG, em julho de 1996.

\begin{tabular}{|c|c|c|c|c|c|c|c|}
\hline \multirow{3}{*}{$\begin{array}{l}\text { Grupo taxonômico } \\
\text { Heteroptera }\end{array}$} & \multirow{2}{*}{\multicolumn{3}{|c|}{ Grupo funcional }} & \multicolumn{2}{|c|}{ Lagoa Carioca } & \multicolumn{2}{|c|}{ Lagoa da Barra } \\
\hline & & & & \multirow{2}{*}{$\begin{array}{c}\text { Litoral } \\
25\end{array}$} & \multirow{2}{*}{$\frac{\text { Limnética }}{-}$} & \multirow{2}{*}{$\begin{array}{c}\text { Litoral } \\
1\end{array}$} & \multirow{2}{*}{$\frac{\text { Limnética }}{-}$} \\
\hline & Notonectidae & & Predador & & & & \\
\hline & Nabidae & & & - & - & 1 & - \\
\hline & Corixidae & & Predador & 3 & - & - & - \\
\hline & Pleidae & & Predador & 7 & - & - & - \\
\hline & Gerridae & & Predador & 1 & - & - & - \\
\hline \multirow[t]{6}{*}{ Coleoptera } & Hydrophilidae & sp 1 & Coletor & 9 & - & - & - \\
\hline & & $\operatorname{sp} 2$ & Coletor & 2 & - & - & - \\
\hline & & sp 3 & Coletor & 4 & - & - & - \\
\hline & & $\mathrm{sp} 4$ & Coletor & 1 & - & - & - \\
\hline & Noteridae & & Predador & 1 & - & - & - \\
\hline & Dytiscidae & & Predador & 1 & - & - & - \\
\hline \multirow[t]{5}{*}{ Ephemeroptera } & Baetidae & sp 1 & Raspador & 29 & - & 1 & - \\
\hline & & sp 2 & Raspador & 2 & - & - & - \\
\hline & & sp 3 & Raspador & 4 & - & - & - \\
\hline & & sp 4 & Raspador & - & - & - & - \\
\hline & & sp 5 & Raspador & 1 & - & - & - \\
\hline \multirow[t]{6}{*}{ Diptera } & Chironomidae & sp 1 & Coletor & 226 & 450 & 15 & 18 \\
\hline & & sp 2 & Coletor & - & 13 & 18 & 30 \\
\hline & & sp 3 & Coletor & - & - & 2 & - \\
\hline & & sp 4 & Coletor & - & - & 1 & - \\
\hline & Ceratopogonida & & Predador & 4 & - & - & - \\
\hline & Chaoboridae & & Predador & - & 2 & - & 11 \\
\hline \multirow[t]{5}{*}{ Trichoptera } & Hydroptilidae & sp 1 & Raspador & 5 & - & - & - \\
\hline & Odontoceridae & sp 1 & Fragmentador & 37 & - & 3 & - \\
\hline & & sp 2 & Fragmentador & 2 & - & - & 1 \\
\hline & & sp 3 & Fragmentador & 39 & - & - & - \\
\hline & Leptoceridae & sp 1 & Fragmentador & 5 & - & - & - \\
\hline \multirow[t]{6}{*}{ Odonata } & Gomphidae & sp 1 & Predador & 29 & - & 4 & - \\
\hline & & $\mathrm{sp} 2$ & Predador & 1 & - & - & - \\
\hline & Libellulidae & sp 1 & Predador & 5 & - & - & - \\
\hline & & $\mathrm{sp} 2$ & Predador & 4 & - & - & - \\
\hline & & sp 3 & Predador & 6 & - & - & - \\
\hline & Coenagrionidae & & Predador & 8 & - & - & - \\
\hline \multirow[t]{2}{*}{ Annelida } & Oligochaeta & & Filtrador & - & 7 & - & 1 \\
\hline & Hirudinea & & Parasita & 3 & 7 & - & 1 \\
\hline \multirow[t]{3}{*}{ Mollusca } & Biomphalaria & & Raspador & 153 & 14 & - & - \\
\hline & Bivalvia & & Filtrador & - & - & - & 1 \\
\hline & Ampullariidae & & Raspador & 2 & - & - & - \\
\hline
\end{tabular}

A diminuição da abundância relativa dos grupos fragmentador e raspador na lagoa da Barra pode ser devida à maior superfície desta em relação à Carioca. Como a lagoa Carioca possui uma área menor, a razão entre a superfície e o perímetro nas duas lagoas é diferente. Dessa forma, o "efeito de borda" torna-se mais diluído na lagoa da Barra, fazendo com que a quantidade de litter cedido pela vegetação ripária, que é a principal fonte de alimento para esses dois grupos, seja proporcionalmente menor nesta em comparação à lagoa Carioca. Além disso, a qualidade do litter que aporta nas lagoas é essencialmente diferente: a lagoa da Barra recebe litter proveniente das plantações de 
Eucalyptus spp. que a margeiam, notadamente de qualidade inferior como item alimentar (Sabará, 1994), principalmente para os organismos raspadores e filtradores.

O grupo dos predadores, assim como os parasitas, habitualmente possuem abundâncias relativamente constantes, já que dependem, diretamente, da presença de outros macroinvertebrados e não dos gradientes de produtividade ou disponibilidade de partículas orgânicas (Vannote et al., 1980).
A similaridade entre os dois ambientes foi calculada através do índice de Sorensen, sendo encontrado o valor de $42 \%$. Essa similaridade relativamente baixa deve-se ao fato de que a lagoa Carioca apresentou um número bem maior de espécies quando comparada à lagoa da Barra. Entretanto, quase todas as espécies presentes na lagoa da Barra também eram encontradas na lagoa Carioca (das 14 espécies presentes na Barra, 10 eram comuns aos dois ambientes).
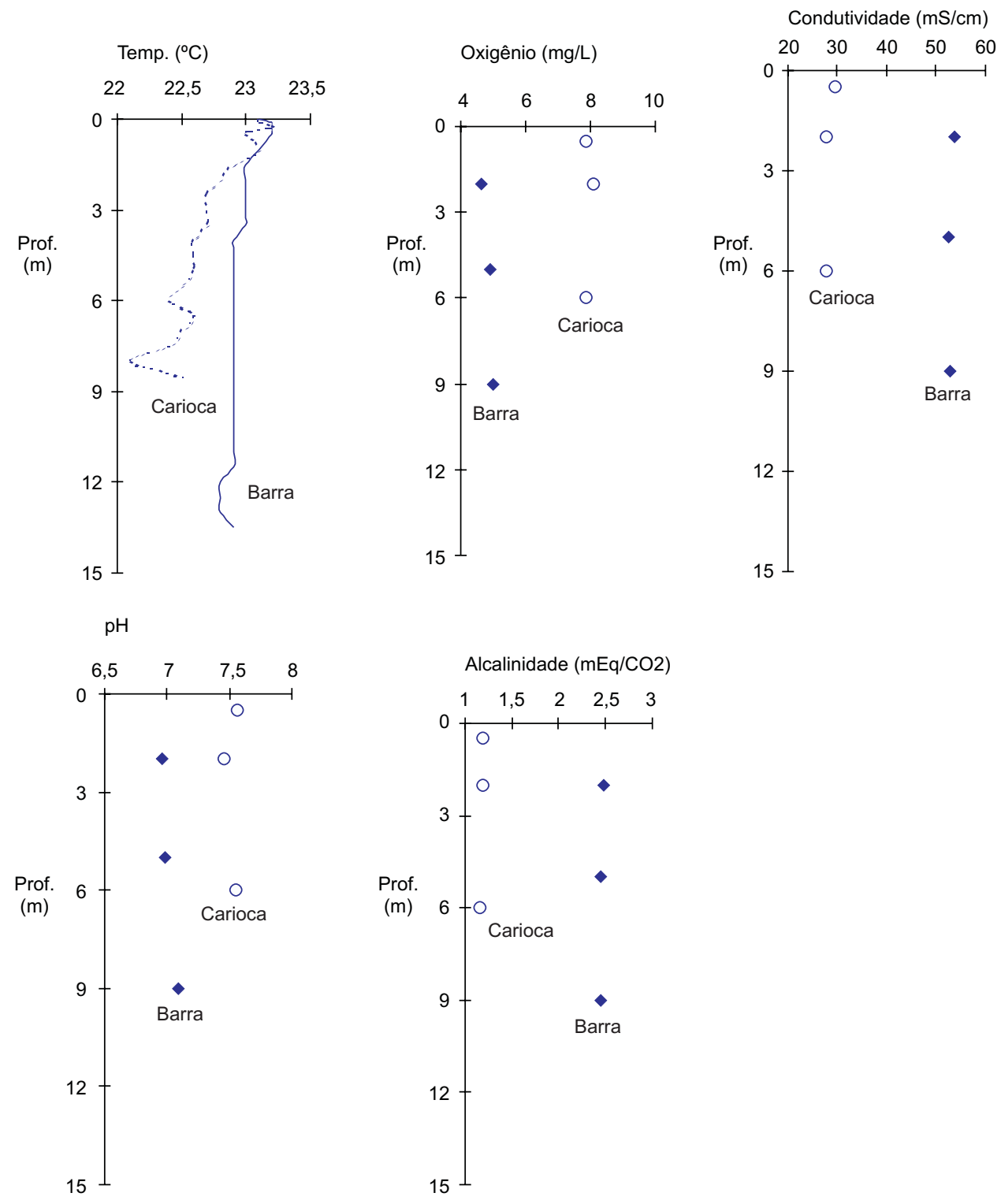

Fig. 1 - Parâmetros físicos e químicos da região limnética das lagoas Carioca e da Barra, obtidos em julho de 1996, Parque Estadual do Rio Doce, MG. 


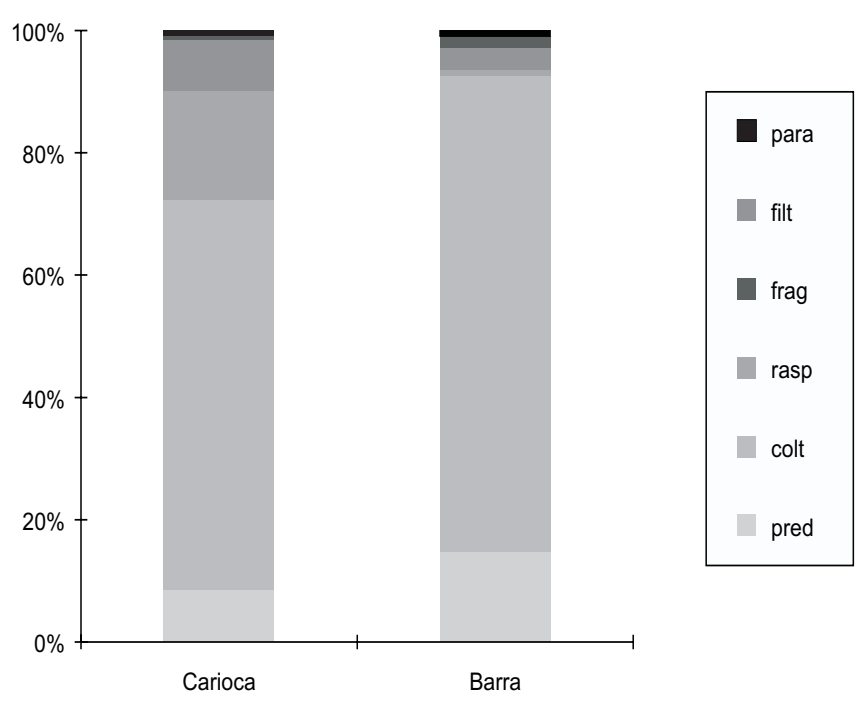

Fig. 2 - Participação de guildas funcionais na composição da comunidade bentônica das lagoas Carioca e da Barra, Parque Estadual do Rio Doce, MG, julho de 1996.

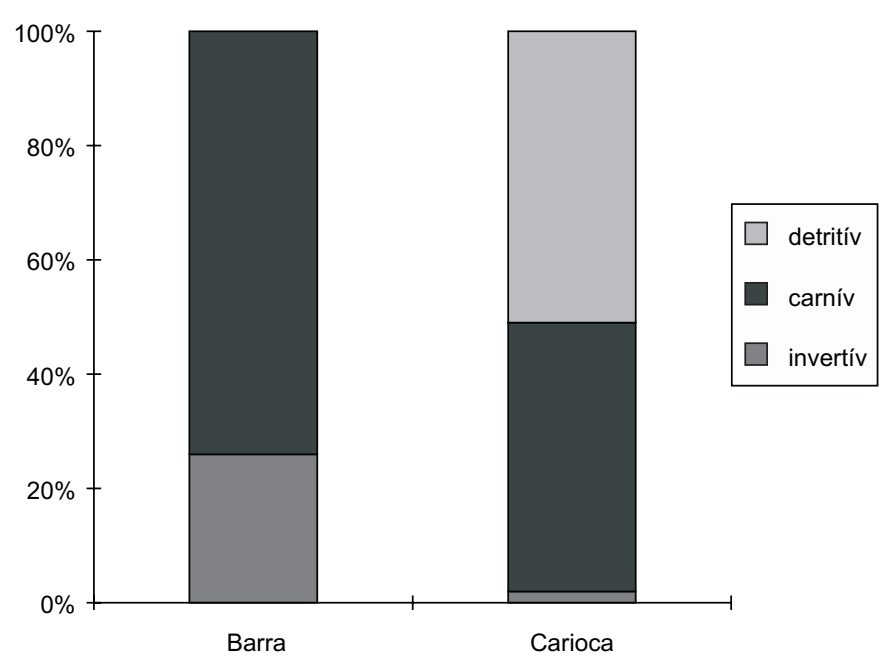

Fig. 3 - Porcentagem de biomassa de peixes por guilda trófica nas lagoas Carioca e da Barra, Parque Estadual do Rio Doce, MG, julho de 1996.

Esse resultado parece indicar que a lagoa da Barra vem sofrendo perdas de espécies, provavelmente devido a perturbações antrópicas iniciadas com o plantio de Eucalyptus spp. em sua bacia.

Além disso, a comunidade de macroinvertebrados da lagoa da Barra vem sofrendo consideravelmente maior pressão de predação por parte da ictiofauna, já que peixes invertívoros, que se alimentam de macroinvertebrados, representam $25 \%$ da biomassa total da ictiofauna nesse ambien- te, enquanto na lagoa Carioca restringem-se a 1,8\% (Fig. 3).

Tais fatores podem ter determinado as diferenças observadas entre a riqueza e a abundância das lagoas em estudo. A manipulação experimental de populações de peixes invertívoros tem mostrado que a biomassa e as taxas de produção da macrofauna bentônica sofrem uma redução severa com o aumento da pressão de predação (Korinková, 1967; Kajak, 1970). 
A pressão de predação pode ter determinado, ainda, a maior riqueza de dípteros encontrada na lagoa da Barra, por efeito de controle da população de alguma espécie competitivamente superior. A supressão dessa espécie ou mesmo uma diminuição em sua população pode estar possibilitando um aumento das populações das espécies adjacentes ou mesmo o aparecimento de novas, aumentando, assim, a riqueza deste grupo de organismos na lagoa.

\section{Limitações do estudo}

Segundo MacArthur \& Wilson (1967), um ambiente com maior área suportaria uma maior quantidade de espécies se comparado a um de menor área. A lagoa da Barra, além de apresentar uma maior superfície, possui um formato dendrítico bem mais complexo que a lagoa Carioca, que é menor, possui formato arredondado e, portanto, apresenta um maior efeito de borda do que esta última. De acordo com estes autores, seria esperado encontrar-se uma maior riqueza, abundância e diversidade na lagoa da Barra, ao contrário do observado. Os resultados encontrados podem ser reflexo do pequeno número de pontos amostrados em ambas as lagoas. Tais coletas puntuais podem estar mascarando o verdadeiro status das comunidades em cada um desses sistemas. A confirmação de uma ou outra tendência será conseguida com a ampliação da rede amostral, abrangendo diferentes microhabitats.

Os resultados obtidos podem ter sido influenciados por alguns fatores, dentre os quais destacase: i) o local das coletas nas lagoas pode ter influenciado o número e mesmo os tipos de organismos associados a cada um dos sistemas; ii) a área litorânea amostrada na lagoa Carioca era praticamente não-impactada, ao contrário da área na lagoa da Barra, que localizava-se em um ancoradouro de barcos de pesca, próxima também de um clube de pesca; iii) além disso, o principal afluente da lagoa da Barra foi barrado a cerca de oito meses, diminuindo, consideravelmente, sua profundidade, segundo os pescadores da região.

Sugere-se um estudo mais aprofundado desses sistemas, levando-se em consideração fatores geomorfológicos, análises de nutrientes e estruturação de hábitats para avaliar quais fatores têm interferido nas comunidades bentônicas dessas lagoas.

\section{CONCLUSÃO}

A comunidade de macroinvertebrados aquáticos mostrou-se mais rica e abundante na lagoa Carioca do que na lagoa da Barra, embora a diversidade em ambas não tenha sido significativamente diferente.

Apesar da lagoa da Barra ser maior e possivelmente abranger maior quantidade de microhábitats, ela vem sendo continuamente submetida a impactos antrópicos que podem estar provocando o empobrecimento de sua fauna bentônica e, conseqüentemente, alterando o funcionamento do ecossistema.

Agradecimentos - Os autores agradecem ao CNPq o apoio financeiro concedido na forma de bolsas.

\section{REFERÊNCIAS BIBLIOGRÁFICAS}

BARBOSA, F. A. R. \& TUNDISI, J. G., 1980, Primary production of phytoplankton and environmental characteristics of a shallow Quarternary lake at Eastern Brasil. Arch. Hydrobiol., 90(2): 139-161.

BARBOSA, F. A. R. \& TUNDISI, J. G., 1989, The influence of temperature variation on the distribution of dissolved oxygen and nutrients. Arch. Hydrobiol., 116(3): 333-349.

CAllisto, M. \& ESTEVES, F. A., 1995, Distribuição da comunidade de macroinvertebrados bentônicos em um lago amazônico impactado por rejeito de bauxita, Lago Batata (Pará, Brasil). Oecologia Brasiliensis. v. 1. Estrutura, Funcionamento e Manejo de Ecossistemas Brasileiros. F. A. Esteves (ed.), pp. 281-291, Programa de Pósgraduação em Ecologia, Instituto de Biologia, Universidade Federal do Rio de Janeiro, Rio de Janeiro.

COLE, G. A., 1983, Textbook of Limnology. 3a ed., C. V. Mosby Company, St. Louis, 401p.

CUMMINS, K. W., WILZBACH, M. A., GATES, D. M., PERRY, J. B. \& TALIAFERRO, W. B., 1989, Shredders and riparian vegetation. Bioscience, 39(1): 24-30.

DEVÁI, G., 1990, Ecological background and importance of the change of chironomid fauna in shallow Lake Balaton. Hidrobiologia, 191: 189-198.

DI GIOVANNI, M. V., GORETTI, E. \& TAMANTI, V., 1996, Macrobenthos in Montedoglio Reservoir, central Italy. Hydrobiologia, 321: 17-28.

ESTEVES, F. A., 1988, Fundamentos de Limnologia. FINEP/ Interciência, 575p.

FUKUARA, H., TORRES, G. \& CLARO, S. M., 1987, The rainy season standing crop of zoobenthos in lakes Dom Helvécio and Jacaré. In: Y. Saijo \& J. Tundisi (eds.) (2º report), Limnological Studies in Central Brazil, Nagoya, $185 \mathrm{p}$. 
KAJAK, Z., 1970, Analysis of the influence of fish on benthos by the method of enclosures. In: Z. Kajak \& A. Hillbricht-Ilkowska (eds.), Productivity Problems of Freshwaters, Warsaw, PWN Polish Scientific Publishers, pp. 781-793.

KORINCOVÁ, J., 1967, Relations between predation pressure of carp, submerged plant development and littoral bottomfauna of Pond Smyslov. Rozpravy Ceskosl. Akad. Ved, Rada Matem, Prirod. Ved., 77(11): 35-62.

MACKERETH, F. J. H., HERON, J. \& TALLING, J. F., 1978, Water analysis: some revised methods for limnologists. Freshwater Biological Association. Scientific Publication $n^{\circ} 36$, Titus Wilson \& Son Ltd. Kendal, 120p.

MCARTHUR, R. H. \& WILSON, E. O., 1967, The theory of island biogeography. Princeton University Press, New Jersey.

MERRIT, R. W. \& CUMMINS, K. W., 1984, An introduction to the aquatic insects of North America. $2^{\mathrm{a}}$ ed., Kendall Hunt Publishing Co., Dubuque, Iowa, 234p.
SABARÁ, M. G., 1994, Avaliação dos impactos do plantio de Eucalyptus spp. sobre dois lagos naturais no médio Rio Doce, MG: propostas de mitigação e manejo. Dissertação de Mestrado, Universidade Federal de Minas Gerais, Brochura.

TATE, C. M. \& HEINY, J. S., 1995, The ordination of benthic invertebrate communities in the South Platte River Basin in relation to environmental factors. Freshwater Biology, 33: 439-454.

VANNOTE, R. L., MINSHALL, G. W., CUMMINS, K. W., SEDELL, J. R. \& CUSHING, C. E., 1980, The river continuum concept. Can. J. Fish. Aquat. Sci., 37: 130-137.

VIEIRA, F., 1994, Estrutura de comunidade e aspectos da alimentação e reprodução dos peixes em dois lagos do médio Rio Doce. Dissertação de Mestrado, Universidade Federal de Minas Gerais, 76p.

WINKLER, L. W., 1888, Die Bertimmung des im wasser gelösten Sauer-stoffs. Ber. dtsch. chem. Ges., 21: 2843 2854. 